\title{
The role of endoscopic ultrasound in children with Pancreatobiliary and gastrointestinal disorders: a single center series and review of the literature
}

\author{
Alessandro Fugazza ${ }^{1}$, Barbara Bizzarri ${ }^{1}$, Federica Gaiani ${ }^{1}$, Marco Manfredi ${ }^{2}$, Alessia Ghiselli ${ }^{1}$, Pellegrino Crafa $^{3}$,
} Maria Clotilde Carra ${ }^{4}$, Nicola de'Angelis ${ }^{5,6}$ and Gian Luigi de'Angelis ${ }^{1 *}$

\begin{abstract}
Background: The role of endoscopic ultrasound (EUS) in the management of pancreatobiliary and digestive diseases is well established in adults, but it remains limited in children. The aim of this study was to evaluate the feasibility, safety, and clinical impact of EUS use in children.
\end{abstract}

Methods: This is a retrospective analysis of a prospectively acquired database of consecutive pediatric ( $<18$ years) patients presenting an indication for EUS for pancreatobiliary and gastrointestinal disorders.

Results: Between January 2010 and January 2016, 47 procedures were performed in 40 children (mean age of 15.1 \pm 4.7 years; range 3-18). The majority of EUS $(n=32 ; 68.1 \%)$ were performed for pancreatobiliary and upper gastrointestinal pathologies, including suspected common bile duct stones (CBDs), acute biliary pancreatitis, recurrent/chronic pancreatitis, cystic pancreatic mass, recurrent hypoglycemia, duodenal polyp, gastric submucosal lesion, and perigastric abscess. In only 2 out of 18 children with suspected CBDs or acute biliary pancreatitis, EUS confirmed CBDs. EUS-guided fine needle aspiration was performed in $3(6.4 \%)$ patients. Fifteen (31.9\%) procedures were performed for lower gastrointestinal tract disorders, including suspected anal Crohn's disease, fecal incontinence, and encopresis. Overall, EUS had a significant impact on the subsequent clinical management in 87. $2 \%$ of patients.

Conclusion: The present findings were consistent with results observed in the current relevant literature and support EUS as a safe and feasible diagnostic and therapeutic tool, which yields a significant clinical impact in children with pancreatobiliary and gastrointestinal disorders.

Keywords: Endoscopic ultrasound, Gastrointestinal disease, Pancreatobiliary disease, Pediatrics

\section{Background}

Endoscopic ultrasound (EUS) and EUS-guided fine needle aspiration (FNA) have been dramatically evolving since their introduction and has become one of the most important techniques for the definitive cytological or histological diagnosis and the management of pancreatobiliary and gastrointestinal (GI) diseases [1-3].

Historically, the primary technique used for the diagnosis and treatment of several pancreatic and biliary diseases in both adults and children was endoscopic

\footnotetext{
* Correspondence: gianluigi.deangelis@unipr.it

${ }^{1}$ Gastroenterology and Endoscopy Unit, University Hospital of Parma, Via

Gramsci 14, 43126 Parma, Italy

Full list of author information is available at the end of the article
}

retrograde cholangiopancreatography (ERCP) $[4,5]$. Recent studies performed in adult populations have identified computed tomography (CT), magnetic resonance cholangiopancreatography (MRCP) and EUS as noninvasive tests that can be used as an alternative to ERCP for pancreatobiliary diseases [6-8] to minimize the risk of associated complications and to eventually prevent unnecessary and invasive diagnostic procedures [4]. In particular, MRCP and EUS are radiation-free imaging exams that are now considered as the best methods for the detection of common bile duct stones (CBDs), yielding the highest diagnostic accuracy $[9,10]$. 
While the role of EUS in adults is well established and widespread, EUS and EUS-FNA in children are supported by limited number of studies, and its indications are restricted compared to adults $[1,3,8,11-17]$. This may be due to multiple factors, including the low incidence of pancreatobiliary disorders and GI tumors in the pediatric population [12], an insufficient awareness among pediatricians, and the limited experience of pediatric endoscopists. Indeed, most EUS procedures in children are performed by adult gastroenterologists because the low number of pediatric EUS procedures does not enable pediatric gastroenterologists to acquire and maintain proficiency in EUS $[12,13]$. However, EUS may have an important clinical impact in children, and efforts should be made to disperse this technique as a valuable diagnostic and therapeutic tool, which minimizes the procedural risks and avoids unnecessary ERCP $[1,15]$.

The present study aims to report the experience of a single high-volume gastroenterology and endoscopy unit in the application of EUS and EUS-FNA in children to further evaluate its feasibility, safety, and clinical impact on pediatric pancreatobiliary and GI disorders. In addition, the present findings are discussed in comparison with current pertinent literature.

\section{Methods}

\section{Study population}

The present study is a retrospective analysis of a prospectively acquired database of consecutive pediatric $(<$ 18 years) patients presenting an indication for EUS or EUS-FNA. All procedures were performed between January 2010 and January 2016 at the Endoscopy Unit of the University of Parma. EUS and EUS-FNA were performed by a senior gastroenterologist (GLdeA) with expertise in both adult and pediatric endoscopy.

Written consent was obtained from both parents or legal guardians, and it included consent for the therapeutic procedures. All data were collected in compliance with the ethical principles stated in the Declaration of Helsinki, and according to the Good Clinical Practice protocols and Privacy Protection Law of the institution.

\section{Techniques}

Upper EUS examinations were performed with patients under deep sedation or general anesthesia performed by a pediatric anesthesiologist depending on the American Society of Anesthesiologists (ASA) classification and the type of procedure. Lower EUS were generally performed without sedation unless specific conditions (e.g., very young age) contraindicated it. A minimum of 10 to $12 \mathrm{~h}$ of fasting were required for upper EUS, whereas 2 enemas were requested before lower EUS.

EUS procedures were performed using different echoendoscopes, including radial echoendoscopes (insertion tube of $13.45 \mathrm{~mm}$, biopsy channel of $2.4 \mathrm{~mm}$; Pentax EG-3670URK, Pentax Hamburg, Germany); linear echoendoscopes (insertion tube of $12.8 \mathrm{~mm}$, biopsy channel of $3.8 \mathrm{~mm}$; Pentax EG-3870UTK, Pentax Hamburg, Germany); linear Slim echoendoscopes (insertion tube of $10.8 \mathrm{~mm}$, biopsy channel $2.8 \mathrm{~mm}$; Pentax EG3270UK, Pentax Hamburg, Germany); or linear ultrasound bronchoscope (insertion tube of $6.3 \mathrm{~mm}$, biopsy channel of $2 \mathrm{~mm}$; Pentax EB1970UK, Pentax Hamburg, Germany) with a Hitachi - Aloka Avius processor (Hitachi, Hamburg, Germany).

The choice of the scope was based on the age and weight of the patient. Specifically, the linear Slim echoendoscope was used for upper echoendoscopy in children younger than 10 years and/or weighing less than $35 \mathrm{~kg}$ (cases 5, 15, 20 and 32; Table 2), while the linear ultrasound bronchoscope was chosen only for the management of case 33, as the child was 4 years old and weighed $13 \mathrm{~kg}$.

Examination of the pancreatic head, biliary tract, gallbladder, and portal regions was performed from the descending duodenum and duodenal bulb; the pancreatic body and tail, and the left lobe of the liver were visualized from the stomach. For lower EUS, the instrument was advanced beyond the rectum, and imaging was performed on slow scope withdrawal after instilling water into the rectum to examine the rectosigmoid junction, rectum and anal canal [14].

EUS-FNA was performed using either a 22- or 25gauge FNA biopsy needle (EchoTip, Wilson-Cook Medical Inc., Winston-Salem, NC) with color Doppler imaging to exclude vessels along the path of the needle. To increase diagnostic accuracy, two or three needle passes were made for solid lesions. Elastography, an indicator of tissue stiffness, was used for differential diagnosis and to address the sampling of solid lesions. One pass was performed for cystic lesions to minimize infection complications. Intravenous antibiotic prophylaxis was administered before EUS-FNA of cystic lesions. Drainage of pancreatic pseudocyst was performed in the most prominent site of the bulge using a 19-gauge needle (EchoTip, Wilson-Cook Medical Inc., Winston-Salem, NC). A 0.035-in. guidewire (Microvasive Endoscopy, Boston Scientific Corp, Galway, Ireland) was inserted through the needle into the pseudocyst under X-ray control. After removal of the needle, a cyst-gastrostome was inserted. Finally, the gastric wall was dilated up to $10 \mathrm{~mm}$ using a wire-guided balloon and a flared-type biflanged metal stent ( $30 \mathrm{~mm}$ length, $10.5 \mathrm{Fr}$, Niti-S Nagi stent, Taewoong Medical Co., Seoul, Korea) was inserted into the cyst cavity.

All adverse events, defined as any event that negatively impacted on the health status of the patient within 30 days from the procedure, were observed via 
outpatient assessments for the first 2 weeks and by weekly telephone contacts with family members and/or referring physicians afterwards.

\section{Study outcomes}

Patient demographics, relevant medical history, initial diagnosis, previous conventional abdominal imaging (ultrasound (US), CT, magnetic resonance imaging (MRI) or MRCP), indication for EUS, specific EUS findings, therapeutic interventions, impact of EUS on the patient's subsequent management, and complications were reviewed and analyzed.

The clinical impact of EUS was scored as [16]:

(0) No impact on diagnosis or management;

(1) Establishment of a definitive diagnosis or exclusion of suspected pathological conditions;

(2) Yield of new, relevant findings, which subsequently altered the patient management strategy.

(3) Yield of relevant findings and EUS-based therapeutic approach.

\section{Pathological examination}

After FNA, the aspirated material was first smeared on a glass slide by the operating endoscopist, taking care that any clotted material was preserved for a cell block. In this case, the material was placed into a container of $10 \%$ neutral-buffered formalin fixative for the creation of a tissue block. Air-dried (for Diff-Quick staining) and fixed smears (fixed immediately in 95\% ethyl alcohol for subsequent Papanicolaou staining) were prepared in an almost equal ratio. All slides were analyzed by an experienced cytopathologist (PC).

\section{Statistical analysis}

Data are reported as the mean and standard deviation or range for continuous variables and as relative frequencies (number and percentages) for categorical variables. The outcome measures (mean values, standard deviation, and ranges) were extracted from the original relevant articles in the analysis of the current literature. Whenever possible, the overall data were analyzed as the sum or weighted mean (and standard deviation).

\section{Results}

During the study period, a total of 2161 EUS were performed in the unit, of which $47(2.17 \%)$ pediatric EUS procedures in 40 patients (18 females, 22 males; mean age of $15.1 \pm 4.7$ years, range $3-18$ ). These included 32 (68.1\%) upper EUS and 15 (31.9\%) lower EUS (Table 1). All EUS procedures were performed in the Endoscopy Unit (not operating room).

The majority of EUS investigated the pancreatobiliary tract $(59.5 \%)$, followed by the rectum (31.9\%), stomach (4.3\%), and duodenum (4.3\%). Overall, 3 (6.4\%) EUS-
Table 1 Study population and indications for EUS

\begin{tabular}{|c|c|}
\hline Children/Procedures [n] & $40 / 47$ \\
\hline Females/Males $[n]$ & $18 / 22$ \\
\hline Age $[y$, range $]$ & $3-18$ \\
\hline$[y$, mean $\pm S D]$ & $15.1 \pm 4.7$ \\
\hline \multicolumn{2}{|l|}{ Indications for EUS [n (\%)] } \\
\hline Upper-GI EUS & $32(68.1)$ \\
\hline - Suspected CBDs & 8 \\
\hline - Acute biliary pancreatitis & 7 \\
\hline - Recurrent/chronic pancreatitis & 4 \\
\hline - Suspected CBDs in patients with UC & 3 \\
\hline - Cystic pancreatic mass & 3 \\
\hline - Recurrent hypoglycemia & 2 \\
\hline - Duodenal polyp & 2 \\
\hline - Pseudocyst drainage & 1 \\
\hline - Gastric submucosal lesion & 1 \\
\hline - Perigastric abscess & 1 \\
\hline Lower-GI EUS & $15(31.9)$ \\
\hline - Suspected anal Crohn's Disease & 12 \\
\hline - Fecal incontinence & 2 \\
\hline - Encopresis & 1 \\
\hline EUS-FNA [n (\%)] & $3(6.4)$ \\
\hline \multicolumn{2}{|l|}{ EUS procedures with sedation [n (\%)] } \\
\hline \multicolumn{2}{|l|}{ oUpper Gl } \\
\hline - Deep sedation & $22(46.8)$ \\
\hline - General anesthesia & $10(21.3)$ \\
\hline \multicolumn{2}{|l|}{ oLower Gl } \\
\hline - No sedation & $14(29.8)$ \\
\hline - Deep sedation & $1(2.1)$ \\
\hline Anesthesia-related adverse events [n (\%)] & 0 \\
\hline \multicolumn{2}{|l|}{ Clinical Impact of EUS [n (\%)] } \\
\hline - Score 0 & $6(12.8)$ \\
\hline - Score 1 & $24(51)$ \\
\hline - Score 2 & $17(36.2)$ \\
\hline Significant impact (score 1 + 2) & $41(87.2)$ \\
\hline
\end{tabular}

FNA were performed with a diagnostic yield of $100 \%$. All 47 procedures were technically successful, and no adverse events, intraoperative or delayed complications occurred. Details of the EUS indications and findings are described below by the organs involved and are shown in Table 2.

\section{Anesthesia}

For upper EUS, deep sedation with propofol was used in $22(46.8 \%)$ procedures, whereas general anesthesia with 
Table 2 EUS procedures by indications and findings

\begin{tabular}{|c|c|c|c|c|c|c|c|c|}
\hline Case & $\begin{array}{l}\text { Age } \\
\text { (y) / } \\
\text { Sex }\end{array}$ & Indication & Comorbidities & $\begin{array}{l}\text { Imagery/ } \\
\text { Diagnostic } \\
\text { Studies Prior } \\
\text { EUS }\end{array}$ & Sedation & EUS Findings & Treatment & Impact \\
\hline 1 & $18 \mathrm{~F}$ & $\begin{array}{l}\text { Suspected } \\
\text { CBDs }\end{array}$ & Nil & US, CT & $\mathrm{DP}$ & Gallstones and CBDs & $\begin{array}{l}\text { Stones extracted at } \\
\text { ERCP; laparoscopic } \\
\text { cholecystectomy }\end{array}$ & 2 \\
\hline 2 & $12 \mathrm{~F}$ & $\begin{array}{l}\text { Suspected } \\
\text { acute biliary } \\
\text { pancreatitis }\end{array}$ & Psoriasis & US, CT & $\mathrm{DP}$ & Normal & Precluded need for ERCP & 1 \\
\hline 3 & $18 \mathrm{M}$ & $\begin{array}{l}\text { Suspected } \\
\text { CBDs }\end{array}$ & $\begin{array}{l}\text { Ulcerative colitis, } \\
\text { sclerosing } \\
\text { cholangitis }\end{array}$ & US, MRI & $\mathrm{DP}$ & Normal & Precluded need for ERCP & 1 \\
\hline 4 & $12 \mathrm{~F}$ & $\begin{array}{l}\text { Suspected } \\
\text { CBDs }\end{array}$ & Nil & US & $\mathrm{DP}$ & Gallstones & $\begin{array}{l}\text { Precluded need for } \\
\text { ERCP; laparoscopic } \\
\text { cholecystectomy }\end{array}$ & 1 \\
\hline 5 & $7 \mathrm{M}$ & $\begin{array}{l}\text { Recurrent } \\
\text { pancreatitis }\end{array}$ & $\begin{array}{l}\text { Klinefelter } \\
\text { syndrome }\end{array}$ & US, MRI & GA & Chronic pancreatitis & Nil & 1 \\
\hline $5 b$ & & $\begin{array}{l}\text { Recurrent } \\
\text { pancreatitis } \\
\text { (1 year later) }\end{array}$ & Nil & $C T$ & GA & Chronic pancreatitis & $\mathrm{Nil}$ & 0 \\
\hline 6 & $14 \mathrm{M}$ & $\begin{array}{l}\text { Acute biliary } \\
\text { pancreatitis }\end{array}$ & Nil & US, CT & $\mathrm{DP}$ & $\begin{array}{l}\text { Gallstones, edematous } \\
\text { pancreatitis }\end{array}$ & $\begin{array}{l}\text { Precluded need for } \\
\text { ERCP; } \\
\text { laparoscopic } \\
\text { cholecystectomy }\end{array}$ & 1 \\
\hline 7 & $15 \mathrm{M}$ & $\begin{array}{l}\text { Suspected } \\
\text { CBDs }\end{array}$ & Nil & US & $\mathrm{DP}$ & Gallstones & $\begin{array}{l}\text { Precluded need for } \\
\text { ERCP; } \\
\text { Laparoscopic } \\
\text { cholecystectomy }\end{array}$ & 1 \\
\hline 8 & $18 \mathrm{M}$ & $\begin{array}{l}\text { Recurrent } \\
\text { hypoglycemia }\end{array}$ & Nil & $C T, M R I$ & DP & $\begin{array}{l}\text { Solid hypoechogenic } \\
\text { hypervascular lesion of } \\
\text { pancreatic tail }\end{array}$ & $\begin{array}{l}\text { FNA with } 25 \mathrm{G} \text {, } \\
\text { diagnosis of insulinomas; } \\
\text { surgical resection }\end{array}$ & 2 \\
\hline 9 & $18 \mathrm{M}$ & $\begin{array}{l}\text { Suspected anal } \\
\text { Crohn's disease }\end{array}$ & $\begin{array}{l}\text { Rectal Crohn's } \\
\text { disease }\end{array}$ & $\begin{array}{l}\text { Colonoscopy, } \\
\text { MRI }\end{array}$ & NS & Normal & $\mathrm{Nil}$ & 0 \\
\hline 10 & $18 \mathrm{M}$ & $\begin{array}{l}\text { Suspected } \\
\text { CBDs }\end{array}$ & Nil & US & $\mathrm{DP}$ & Gallstones & $\begin{array}{l}\text { Precluded need for } \\
\text { ERCP; } \\
\text { laparoscopic } \\
\text { cholecystectomy }\end{array}$ & 1 \\
\hline 11 & $18 \mathrm{M}$ & $\begin{array}{l}\text { Suspected anal } \\
\text { Crohn's disease }\end{array}$ & $\begin{array}{l}\text { Rectal Crohn's } \\
\text { disease }\end{array}$ & Colonoscopy, CT & NS & Trans-sphincteric fistula & Biologic therapy & 2 \\
\hline $11 b$ & & $\begin{array}{l}\text { Control after } \\
6 \text { months of } \\
\text { therapy }\end{array}$ & Nil & EUS & NS & Partial remission & Biologic therapy & 2 \\
\hline $11 \mathrm{c}$ & & $\begin{array}{l}\text { Control after } \\
1 \text { year of } \\
\text { therapy }\end{array}$ & Nil & EUS & NS & Remission & Stop of biologic therapy & 2 \\
\hline 12 & $18 \mathrm{~F}$ & $\begin{array}{l}\text { Suspected } \\
\text { CBDs }\end{array}$ & Nil & US, MRI & $\mathrm{DP}$ & Gallstones & $\begin{array}{l}\text { Precluded need for } \\
\text { ERCP; } \\
\text { laparoscopic } \\
\text { cholecystectomy }\end{array}$ & 1 \\
\hline 13 & $16 \mathrm{M}$ & $\begin{array}{l}\text { Suspected } \\
\text { Crohn's disease }\end{array}$ & $\begin{array}{l}\text { Rectal Crohn's } \\
\text { disease }\end{array}$ & Colonoscopy, CT & NS & Normal & $\mathrm{Nil}$ & 0 \\
\hline 14 & $13 \mathrm{~F}$ & $\begin{array}{l}\text { Recurrent } \\
\text { pancreatitis }\end{array}$ & Celiac disease & US, CT & DP & Chronic pancreatitis & Nil & 1 \\
\hline 15 & $9 \mathrm{M}$ & $\begin{array}{l}\text { Suspected } \\
\text { acute biliary } \\
\text { pancreatitis }\end{array}$ & Nil & US, CT & $\mathrm{DP}$ & $\begin{array}{l}\text { Gallstones edematous } \\
\text { pancreatitis }\end{array}$ & $\begin{array}{l}\text { Precluded need for } \\
\text { ERCP; } \\
\text { laparoscopic } \\
\text { cholecystectomy }\end{array}$ & 1 \\
\hline 16 & $16 \mathrm{~F}$ & & & US & GA & & FNA with $22 \mathrm{G}$ & 1 \\
\hline
\end{tabular}


Table 2 EUS procedures by indications and findings (Continued)

\begin{tabular}{|c|c|c|c|c|c|c|c|c|}
\hline Case & $\begin{array}{l}\text { Age } \\
\text { (y) / } \\
\text { Sex }\end{array}$ & Indication & Comorbidities & $\begin{array}{l}\text { Imagery/ } \\
\text { Diagnostic } \\
\text { Studies Prior } \\
\text { EUS }\end{array}$ & Sedation & EUS Findings & Treatment & Impact \\
\hline & & $\begin{array}{l}\text { Cystic } \\
\text { pancreatic } \\
\text { mass }\end{array}$ & $\begin{array}{l}\text { Takayasu arteritis } \\
\text { Hashimoto } \\
\text { thyroiditis }\end{array}$ & & & $\begin{array}{l}\text { Voluminous head pancreatic } \\
\text { cysts }\end{array}$ & (serous cystadenoma) & \\
\hline $16 b$ & & $\begin{array}{l}\text { Acute } \\
\text { pancreatitis }\end{array}$ & Nil & $C T$ & GA & $\begin{array}{l}\text { Compression of CBD and } \\
\text { Wirsung duct }\end{array}$ & Whipple resection & 2 \\
\hline 17 & $12 \mathrm{M}$ & $\begin{array}{l}\text { Fecal } \\
\text { incontinence }\end{array}$ & $\begin{array}{l}\text { Surgery for } \\
\text { Hirschsprung } \\
\text { disease }\end{array}$ & MRI & NS & $\begin{array}{l}\text { Interruption of internal anal } \\
\text { sphincter }\end{array}$ & $\begin{array}{l}\text { Symptomatic } \\
\text { management }\end{array}$ & 1 \\
\hline 18 & $18 \mathrm{~F}$ & $\begin{array}{l}\text { Suspected anal } \\
\text { Crohn's disease }\end{array}$ & $\begin{array}{l}\text { Ileo-colonic } \\
\text { Crohn's disease }\end{array}$ & Colonoscopy, CT & NS & Normal & $\mathrm{Nil}$ & 0 \\
\hline 19 & $12 \mathrm{M}$ & $\begin{array}{l}\text { Suspected anal } \\
\text { Crohn's disease }\end{array}$ & $\begin{array}{l}\text { Colonic Crohn's } \\
\text { disease }\end{array}$ & Colonoscopy & NS & Extra sphincteric fistula & Biologic therapy & 2 \\
\hline $19 b$ & & $\begin{array}{l}\text { Control after } \\
6 \text { months of } \\
\text { therapy }\end{array}$ & Nil & EUS & NS & Remission & Biologic therapy & 2 \\
\hline 20 & $9 \mathrm{~F}$ & $\begin{array}{l}\text { Duodenal } \\
\text { polyp }\end{array}$ & Nil & $\begin{array}{l}\text { EGD, MRI, } \\
\text { PET with Ga- } \\
\text { DOTATOC }\end{array}$ & GA & $\begin{array}{l}\text { Hypoechoic, hypervascular } \\
\text { lesion originate in the III layer, } \\
\text { infiltrate the IV }\end{array}$ & $\begin{array}{l}\text { Surgical resection } \\
\text { (NET G2) }\end{array}$ & 2 \\
\hline $20 \mathrm{~b}$ & & $\begin{array}{l}\text { Follow up after } \\
\text { surgery }\end{array}$ & Nil & $\begin{array}{l}\text { MRI, CT, } \\
\text { PET with Ga- } \\
\text { DOTATOC }\end{array}$ & GA & Normal & $\mathrm{Nil}$ & 0 \\
\hline 21 & $13 \mathrm{~F}$ & $\begin{array}{l}\text { Suspected } \\
\text { acute biliary } \\
\text { pancreatitis }\end{array}$ & Nil & US, MRI & DP & $\begin{array}{l}\text { Gallstones, } \\
\text { acute necrotizing pancreatitis }\end{array}$ & $\begin{array}{l}\text { Precluded need for } \\
\text { ERCP; } \\
\text { laparoscopic } \\
\text { cholecystectomy }\end{array}$ & 1 \\
\hline $21 b$ & & $\begin{array}{l}\text { Abdominal } \\
\text { pain }\end{array}$ & Nil & CT & GA & Pancreatic pseudocyst & $\begin{array}{l}\text { Transgastric drainage } \\
\text { with metallic stent }\end{array}$ & 2 \\
\hline 22 & $12 \mathrm{~F}$ & $\begin{array}{l}\text { Fecal } \\
\text { incontinence }\end{array}$ & $\begin{array}{l}\text { Surgery for } \\
\text { Hirschsprung } \\
\text { disease }\end{array}$ & MRI & NS & $\begin{array}{l}\text { Interruption of internal anal } \\
\text { sphincter }\end{array}$ & $\begin{array}{l}\text { Symptomatic } \\
\text { management }\end{array}$ & 1 \\
\hline 23 & $15 \mathrm{M}$ & $\begin{array}{l}\text { Suspected } \\
\text { CBDs }\end{array}$ & $\begin{array}{l}\text { Ulcerative colitis } \\
\text { sclerosing } \\
\text { cholangitis }\end{array}$ & US, MRI & DP & Normal & Precluded need for ERCP & 1 \\
\hline 24 & $17 \mathrm{~F}$ & $\begin{array}{l}\text { Suspected anal } \\
\text { Crohn's disease }\end{array}$ & $\begin{array}{l}\text { Colonic Crohn's } \\
\text { disease }\end{array}$ & $\begin{array}{l}\text { Colonoscopy, } \\
\text { MRI }\end{array}$ & NS & $\begin{array}{l}\text { Abscess with extra sphincteric } \\
\text { fistula }\end{array}$ & Surgical intervention & 2 \\
\hline 25 & $18 M$ & $\begin{array}{l}\text { Suspected } \\
\text { CBDs }\end{array}$ & $\begin{array}{l}\text { Ulcerative Colitis } \\
\text { sclerosing } \\
\text { cholangitis }\end{array}$ & US, MRI & DP & Normal & Precluded need for ERCP & 1 \\
\hline 26 & $17 \mathrm{M}$ & $\begin{array}{l}\text { Suspected } \\
\text { CBDs }\end{array}$ & Nil & US & $\mathrm{DP}$ & Gallstones & $\begin{array}{l}\text { Precluded need for } \\
\text { ERCP; } \\
\text { laparoscopic } \\
\text { cholecystectomy }\end{array}$ & 1 \\
\hline 27 & $18 \mathrm{M}$ & $\begin{array}{l}\text { Suspected } \\
\text { Crohn's anal } \\
\text { disease }\end{array}$ & $\begin{array}{l}\text { Ileo-colonic } \\
\text { Crohn's disease }\end{array}$ & Colonoscopy, CT & NS & $\begin{array}{l}\text { Abscess with extra sphincteric } \\
\text { fistula }\end{array}$ & Surgical intervention & 2 \\
\hline 28 & $12 \mathrm{M}$ & $\begin{array}{l}\text { Recurrent } \\
\text { pancreatitis }\end{array}$ & Nil & MRI & DP & Chronic pancreatitis & $\mathrm{Nil}$ & 1 \\
\hline 29 & $17 \mathrm{M}$ & $\begin{array}{l}\text { Suspected } \\
\text { acute biliary } \\
\text { pancreatitis }\end{array}$ & Nil & US, CT & $\mathrm{DP}$ & $\begin{array}{l}\text { Gallstones, edematous } \\
\text { pancreatitis }\end{array}$ & $\begin{array}{l}\text { Precluded need for } \\
\text { ERCP; } \\
\text { laparoscopic } \\
\text { cholecystectomy }\end{array}$ & 1 \\
\hline 30 & $18 \mathrm{M}$ & $\begin{array}{l}\text { Suspected } \\
\text { Crohn's anal } \\
\text { disease }\end{array}$ & Nil & $\begin{array}{l}\text { Colonoscopy, } \\
\text { MRI }\end{array}$ & NS & $\begin{array}{l}\text { Abscess with extra sphincteric } \\
\text { fistula }\end{array}$ & Surgical intervention & 2 \\
\hline 31 & $14 \mathrm{~F}$ & & Nil & $\mathrm{EGD}$ & $\mathrm{DP}$ & Lipoma & $\mathrm{Nil}$ & 1 \\
\hline
\end{tabular}


Table 2 EUS procedures by indications and findings (Continued)

\begin{tabular}{|c|c|c|c|c|c|c|c|c|}
\hline Case & $\begin{array}{l}\text { Age } \\
(y) / \\
\text { Sex }\end{array}$ & Indication & Comorbidities & $\begin{array}{l}\text { Imagery/ } \\
\text { Diagnostic } \\
\text { Studies Prior } \\
\text { EUS }\end{array}$ & Sedation & EUS Findings & Treatment & Impact \\
\hline & & $\begin{array}{l}\text { Gastric } \\
\text { subepithelial } \\
\text { lesions }\end{array}$ & & & & & & \\
\hline 32 & $9 \mathrm{~F}$ & $\begin{array}{l}\text { Suspected } \\
\text { acute biliary } \\
\text { pancreatitis }\end{array}$ & Nil & US, MRI & GA & Normal & Precluded need for ERCP & 1 \\
\hline 33 & $4 \mathrm{~F}$ & $\begin{array}{l}\text { Cystic } \\
\text { pancreatic } \\
\text { mass on US }\end{array}$ & Nil & MRI & GA & Pancreatic pseudocyst & $\begin{array}{l}\text { Surgery in urgency for } \\
\text { traumatic rupture }\end{array}$ & 1 \\
\hline 34 & $18 \mathrm{~F}$ & $\begin{array}{l}\text { Suspected } \\
\text { CBDs }\end{array}$ & Nil & US & $\mathrm{DP}$ & Gallstones & $\begin{array}{l}\text { Precluded need for } \\
\text { ERCP; } \\
\text { laparoscopic } \\
\text { cholecystectomy }\end{array}$ & 1 \\
\hline 35 & $18 \mathrm{~F}$ & $\begin{array}{l}\text { Perigastric } \\
\text { abscess at US }\end{array}$ & $\begin{array}{l}\text { PEG, } \\
\text { holoprosencephaly }\end{array}$ & EGD, US & GA & Perigastric abscess & Surgical drainage & 0 \\
\hline 36 & $18 \mathrm{M}$ & $\begin{array}{l}\text { Recurrent } \\
\text { hypoglycemia }\end{array}$ & Nil & MRI & $\mathrm{DP}$ & $\begin{array}{l}\text { Solid hypoechogenic } \\
\text { hypervascular lesion of uncinate } \\
\text { process }\end{array}$ & $\begin{array}{l}\text { FNA with } 25 \mathrm{G} \\
\text { (diagnosis of } \\
\text { insulinomas); } \\
\text { Medical therapy }\end{array}$ & 2 \\
\hline 37 & $18 \mathrm{~F}$ & $\begin{array}{l}\text { Suspected } \\
\text { acute biliary } \\
\text { pancreatitis }\end{array}$ & Nil & US, CT & $\mathrm{DP}$ & $\begin{array}{l}\text { Gallstones, edematous } \\
\text { pancreatitis }\end{array}$ & $\begin{array}{l}\text { Precluded need for } \\
\text { ERCP; } \\
\text { laparoscopic } \\
\text { cholecystectomy }\end{array}$ & 1 \\
\hline 38 & $16 \mathrm{M}$ & $\begin{array}{l}\text { Suspected } \\
\text { CBDs }\end{array}$ & Nil & US & $\mathrm{DP}$ & Gallstones and CBDs & Stones extracted at ERCP & 2 \\
\hline 39 & $3 F$ & Encopresis & $\begin{array}{l}\text { Sacrococcygeal } \\
\text { Yolk Sac Tumor }\end{array}$ & MRI & $\mathrm{DP}$ & Pararectal lesion & $\begin{array}{l}\text { Surgical intervention } \\
\text { (recurrent disease) }\end{array}$ & 2 \\
\hline 40 & $13 \mathrm{M}$ & $\begin{array}{l}\text { Suspected } \\
\text { Crohn's anal } \\
\text { disease }\end{array}$ & $\begin{array}{l}\text { Ilelonic Crohn's } \\
\text { disease }\end{array}$ & $\begin{array}{l}\text { Colonoscopy, } \\
\text { MRI }\end{array}$ & NS & Perianal abscess & Surgical intervention & 2 \\
\hline
\end{tabular}

$C B D$ indicates common bile duct; $C B D$ s indicates common bile duct stones; $C T$ indicates computerized tomography; $D P$ indicates deep sedation; $E G D$ indicates Esophagogastroduodenoscopy; $E R C P$ indicates endoscopic retrograde cholangiopancreatography; $E U S$ indicates endoscopic ultrasound; $F$ indicates female; $F N A$ indicates fine needle aspiration; GA indicates general anesthesia; $M$ indicates male; $M R I$ indicates magnetic resonance imaging; NET indicates neuroendocrine tumor; NS indicates non sedation; PEG indicates percutaneous endoscopic gastrostomy; PET with Ga-DOTATOC indicates Gallium-68-somatostatin receptor positron emission tomography; US indicates ultrasound

endotracheal intubation was performed in 10 (21.3\%) procedures. For lower EUS, 14 (29.8\%) procedures were managed without sedation and only one procedure (2.1\%) was approached with deep sedation due to the very young age of the patient and the presence of comorbidity (case n. 39). No sedation- or anesthesiarelated complications occurred.

\section{Pancreatobiliary system}

The pancreatobiliary system was endosonographically evaluated in 28 (59.6\%) procedures, including 3(6.4\%) EUS-FNA. The indications for EUS were: suspected CBDs $(n=8,28.6 \%)$, suspected acute biliary pancreatitis $(n=7,25 \%), \quad$ recurrent/chronic pancreatitis $(n=4$, $14.3 \%)$, suspected CBDs in patients with ulcerative colitis $(n=3,10.7 \%)$, cystic pancreatic mass $(n=3,10.7 \%)$, recurrent hypoglycemia $(n=2,7.1 \%)$, and drainage of pseudocyst $(n=1,3.6 \%)$. EUS for suspected CBDs was performed in the presence of cholestatic liver biochemistry with imaging suggestive of gallstones by US and MRI. Out of the 8 cases performed, 2 patients' EUS showed the presence of CBDs, which were retrieved by ERCP during the same sedation session.

In the 7 cases of clinically and radiologically suspected acute biliary pancreatitis, EUS showed normal pancreatic parenchyma in $2 / 7$ (28.6\%) patients; endosonographic criteria for acute edematous pancreatitis with gallstones without CBDs in 4/7 (57.1\%) patients; and acute necrotizing pancreatitis with gallstones without CBDs in one patient (14.3\%). After 6 weeks, this latter patient (case n. 21) developed a voluminous pseudocyst with recurrent abdominal pain. Transgastric drainage was performed and a metallic stent was implanted. After an additional 6 weeks, CT imaging confirmed the cyst resolution and the stent was removed endoscopically. 
In the 3 cases of recurrent pancreatitis (case n. 5, 14, 28), EUS showed endosonographic criteria for chronic pancreatitis without requiring further interventions. One of these patients presented with another episode of acute pancreatitis one year later. EUS was performed and showed the same results.

In 3 patients affected by ulcerative colitis with the presence of cholestatic liver biochemistry (case n. 3, 23, 25), MRI showed intrahepatic sclerosing cholangitis and CBDs were suspected. At the EUS examination, no stones were revealed and no ERCP was performed.

In the 2 patients with recurring episodes of hypoglycemia, EUS detected a solid hypoechogenic, hypervascular lesion with distinct boundaries of the uncinate process in one patient (case n. 36) and of the tail in the other patient (case n. 8), with lower elasticity values compared to a healthy pancreas. EUS-FNA was performed with a $25 \mathrm{G}$ needle and a diagnosis of insulinoma was made in both cases (Fig. 1a-d, Fig. 2). Medical therapy was started in the first patient due to the advanced disease, whereas surgical resection was planned for the second patient.

The 2 patients with cystic pancreatic masses on US were referred to our center for EUS (cases n. 16, 33). In one case, EUS-FNA was performed. The endosonographic characteristics and pancreatic cyst fluid analysis were suggestive of a voluminous serous cystadenoma of the pancreatic head. EUS was repeated after 1 year due to acute pancreatitis, which demonstrated an increase in the cyst size with compression of the common bile and Wirsung ducts. Consequently, the patient underwent successful Whipple's resection. In the second case, EUS diagnosed a pancreatic pseudocyst. A linear ultrasound

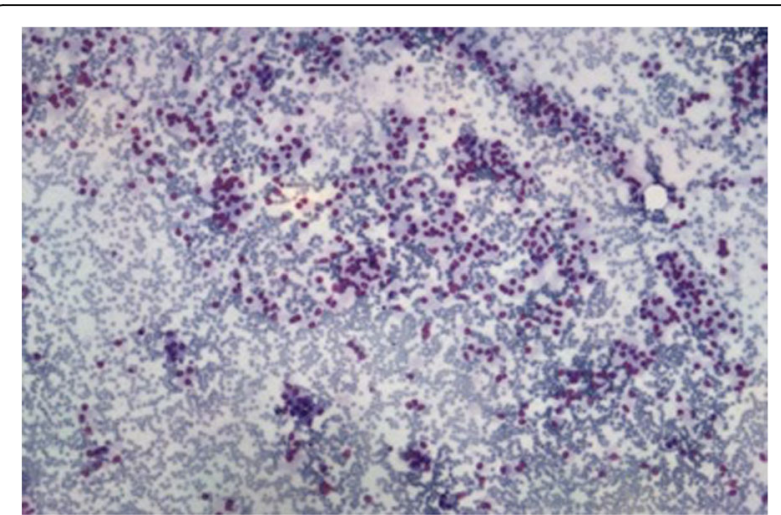

Fig. 2 Fine needle aspirate showed single dispersed, uniform neoplastic cells, which rarely collect in clusters. The neoplastic cells appear round to oval and bland with eccentrically located nuclei (plasmacytoid appearance). No mitosis and no necrosis are observed in the background. Hematoxylin-Eosin 4x magnification

bronchoscope was used only in this child (case n. 33) due to the very young age of the patient. Endoscopic drainage was planned but not performed because an emergency surgery was required for the rupture of the pseudocyst due to an abdominal trauma. The postoperative period was uneventful.

Among patients who underwent EUS for suspected CBDs or biliary pancreatitis, 12 of them (cases 1, 4, 6, 7, 10, 12, 15, 21, 26, 29, 34, 37; Table 2) avoided ERCP and underwent laparoscopic elective cholecystectomy, with a 4-week surgical follow-up. Five other cases (cases 2, 3, 23, 25, 32; Table 2) avoided ERCP, but those presenting with comorbidities affecting the biliary duct (e.g., sclerosing cholangitis, cases $3,23,25$ ) were followed-up by

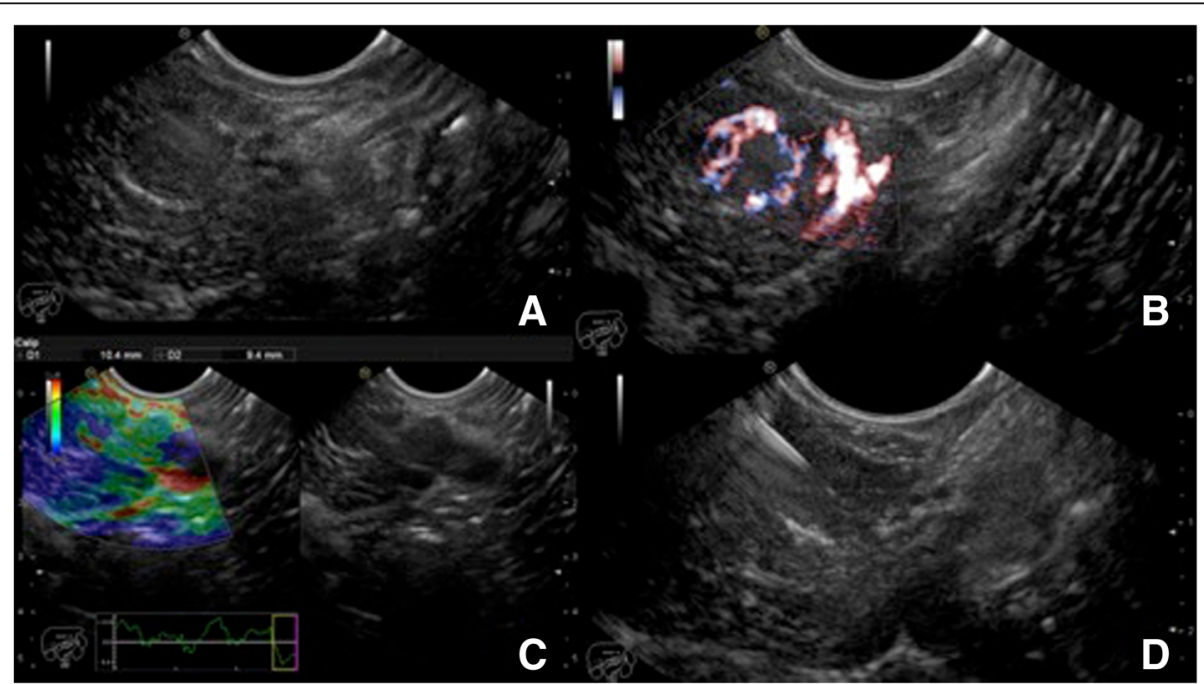

Fig. 1 a: Endoscopic ultrasound (EUS) detection of solid hypoechogenic lesion with distinct boundaries in the tail of the pancreas. b: Color Doppler application revealing a hypervascular lesion. c: Elastography application revealing lower elasticity values compared to healthy pancreas. d: EUS-guided Fine Needle Aspiration (FNA) with a $25 \mathrm{G}$ needle, yielding the final diagnosis of insulinomas 
abdominal ultrasound and/or MRI and biology tests according to the ACG guidelines 2015; the two patients who underwent EUS for pancreatitis in absence of other pancreatobiliary comorbidities were followed-up clinically and with biology tests at 6 and 12 months, including complete hepatic function tests, CRP and lipase, documenting a complete normalization of both clinic and biology.

\section{Upper GI tract}

The indications for upper GI tract EUS included: characterization of duodenal polyp, gastric submucosal lesion, and perigastric abscess.

In one patient (case n. 20), bioptic specimens were suspicious for a neuroendocrine tumor (NET) of the posterior wall of the duodenal bulb. A duodenal hypoechoic, round-shaped, hypervascular lesion that originated in the submucosa and infiltrated the muscularis propria was detected. Surgical resection was required. Histology confirmed the diagnosis of NET G2, according to the 2010 World Health Organization classification [18]. Follow-up was scheduled every 6 months; CT, Gallium-68-somatostatin receptor positron emission tomography (PET with Ga-DOTATOC), EUS and plasmatic chromogranin A levels were all negative.

The second patient (case n. 31) who received upper GI EUS was referred for an endosonographic evaluation of a gastric subepithelial lesion. EUS with contrast enhanced showed a hyperechogenic submucosal lesion with regular margins suggestive of a lipoma was observed. The aspect of the mucosa was normal.

The third patient (case n. 35) presented with holoprosencephaly and a percutaneous endoscopic gastrostomy (PEG). The patient developed a peristomal infection with a perigastric abscess. EUS was performed to characterize and drain the lesion, which was not possible due to its location and surgery was required.

\section{Lower GI tract}

Fifteen lower EUS procedures (31.9\%) were performed. Nine children had suspected anal Crohn's disease. Normal endosonographic findings were found in 3/9 cases. Three children (cases n. 24, 27, 30) had abscesses with extra sphincteric fistulas, whereas 1 patient had a perianal abscess (case n. 40). These four patients were treated surgically. In 1 child (case n. 11), a transsphincteric fistula was observed and medical therapy was started. EUS was performed bi-yearly to evaluate the response to therapy. Six months after the beginning of therapy, residual inflammation was demonstrated, but at 1 year a complete resolution was obtained.

In 1 child (case n. 19), EUS showed an extrasphincteric fistula and medical therapy was started. Six months later, EUS demonstrated a complete resolution.
In 2 children (cases $n .17,22$ ) the indication for EUS was fecal incontinence after surgery for Hirschsprung disease. EUS showed an interruption of the internal anal sphincter.

The last case (case n. 39) was a child with encopresis and previous surgery for sacrococcygeal yolk sac tumor. EUS showed a pararectal lesion suspicious for recurrent disease. The patient underwent surgery and a histological examination confirmed the diagnosis.

\section{Clinical impact of EUS}

According to the predefined criteria [16], 6 (12.8\%) EUS procedures yielded no further information compared to previous imaging results (classified as score 0 ). Twentyfour $(51 \%)$ procedures were classified as score 1 because EUS established a definitive diagnosis or excluded a suspected pathological condition, thereby avoiding more invasive procedures. In the remaining 16 (34.1\%) cases, EUS showed specific findings that allowed for targeted therapy (classified as score 2).

In one case (2.1\%) EUS yielded significant results and allowed endoscopic therapy with EUS-guided cystgastrostome placement (classified as score 3). Overall, EUS had a positive clinical impact (score $1+2+3$ ) in 41 $(87.2 \%)$ procedures, affecting the subsequent clinical management.

According to the EUS findings, the therapeutic management was established as: medical therapy in the $5 \mathrm{pa}$ tients affected by Crohn's disease and in one patient with a neuroendocrine tumor; surgical intervention in 8 patients; and endoscopic therapy in 3 patients.

\section{Pediatric EUS and EUS-FNA cases in the literature}

Table 3 shows the most relevant studies in the literature evaluating the application of EUS and EUS-FNA in pediatric populations. From 1998 to 2016, 10 studies [1, $3,8,11-17$ ] were published with a total of 413 patients and 456 EUS (of which 69 (15.1\%) were EUS-FNA) evaluated. Five studies were performed in the USA [11-13, 15, 17], 3 in Europe [1, 14, 16] and 2 in Asia [3, 8]. The main indication for EUS was the investigation of the pancreatobiliary tract in $324(71.1 \%)$ cases. EUS-related complications were reported in only 3 studies $[1,8,11]$, with an incidence rate ranging between $1.96 \%$ and $3.8 \%$. Only $7 / 10$ studies $[1,3,8,11,12,15,16]$ evaluated the clinical impact of EUS, and these reported a positive impact in an average of $73.5 \%$ (range 35.5-98\%) of cases.

\section{Discussion}

The present study illustrates the experience of a single high-volume endoscopic center in the application of EUS and EUS-FNA for several pediatric pancreatobiliary and GI pathologies. The case series included 47 procedures that were all technically successful, uneventful, 
Table 3 Summary of the current relevant literature and comparison with the present results

\begin{tabular}{|c|c|c|c|c|c|c|c|c|c|c|c|}
\hline \multirow[b]{2}{*}{ Study } & \multirow[b]{2}{*}{$\begin{array}{l}\text { No. } \\
\text { patients }\end{array}$} & \multirow[b]{2}{*}{$\begin{array}{l}\text { No. } \\
\text { EUS }\end{array}$} & \multirow[b]{2}{*}{$\begin{array}{l}\text { Time frame } \\
\text { (No. years) }\end{array}$} & \multirow[b]{2}{*}{$\begin{array}{l}\text { Age }(y) \text {, range } \\
\text { (mean) }\end{array}$} & \multicolumn{6}{|c|}{ Indications no, (\%) } & \multirow[b]{2}{*}{$\begin{array}{l}\text { EUS-FNA } \\
\text { no, (\%) }\end{array}$} \\
\hline & & & & & Pancreatobiliary & Rectum & Stomach & Esophagus & Duodenum & Other & \\
\hline $\begin{array}{l}\text { Roseau et al. } \\
1998 \text { [14] }\end{array}$ & 18 & 23 & 7 & $\begin{array}{l}4-16 \\
(12)\end{array}$ & $8(34.8)$ & $6(26.1)$ & $6(26.1)$ & $1(4.3)$ & $1(4.3)$ & $1(4.3)$ & 0 \\
\hline $\begin{array}{l}\text { Varadarajulu et al. } \\
2005 \text { [15] }\end{array}$ & 14 & 15 & 3 & 5-17 (13) & $15(100)$ & 0 & 0 & 0 & 0 & 0 & $3(20)$ \\
\hline $\begin{array}{l}\text { Cohen et al. } 2008 \\
\text { [3] }\end{array}$ & 32 & 32 & 6 & $1.5-18(12)$ & $19(59.4)$ & $2(6.3)$ & $2(6.3)$ & $8(25)$ & $1(3.1)$ & 0 & $7(21.9)$ \\
\hline $\begin{array}{l}\text { Bjerring et al. } \\
2008 \text { [16] }\end{array}$ & 18 & 18 & 16 & $0.5-15(12)$ & $11(61.1)$ & 0 & $3(16.7)$ & 0 & 0 & $\begin{array}{l}4 \\
(22.2)\end{array}$ & 0 \\
\hline $\begin{array}{l}\text { Attila et al. } 2009 \\
\text { [13] }\end{array}$ & 38 & 40 & 7 & $3-17(13.5)$ & $25(62.5)$ & $1(2.5)$ & $6(15)$ & $1(2.5)$ & 0 & $\begin{array}{l}7 \\
(17.5)\end{array}$ & $12(30)$ \\
\hline $\begin{array}{l}\text { Al-Rashdan et al. } \\
2010 \text { [12] }\end{array}$ & 56 & 58 & 8 & 4-18 (16) & $42(72.4)$ & $4(6.9)$ & $1(1.7)$ & $1(1.7)$ & 0 & $\begin{array}{l}10 \\
(17.2)\end{array}$ & $15(25.9)$ \\
\hline $\begin{array}{l}\text { Rosen et al. } 2010 \\
\text { [17] }\end{array}$ & 25 & 42 & 5 & NA (14) & 0 & $\begin{array}{l}42 \\
(100)\end{array}$ & 0 & 0 & 0 & 0 & 0 \\
\hline $\begin{array}{l}\text { Scheers et al. } \\
2015 \text { [1] }\end{array}$ & 48 & 52 & 14 & 2-17 (12) & $52(100)$ & 0 & 0 & 0 & 0 & 0 & $12(23.1)$ \\
\hline $\begin{array}{l}\text { Gordon et al. } \\
2015 \text { [11] }\end{array}$ & 43 & 51 & 6 & $4-18(14.5)$ & $34(66.7)$ & $1(1.9)$ & $6(11.8)$ & 0 & 0 & $\begin{array}{l}10 \\
(19.6)\end{array}$ & $13(25.5)$ \\
\hline $\begin{array}{l}\text { Mahajan et al. } \\
2016[8]\end{array}$ & 121 & 125 & 8 & $3-18(15.2)$ & $118(94.4)$ & 0 & $2(1.6)$ & 0 & 0 & $5(4)$ & $7(5.6)$ \\
\hline $\begin{array}{l}\text { TOTAL } \\
\text { (sum or } \\
\text { weighted mean) }\end{array}$ & 413 & 456 & 8 & $0.5-18(14)$ & $324(71.1)$ & $\begin{array}{l}56 \\
(12.3)\end{array}$ & $26(5.7)$ & $11(2.4)$ & $2(0.4)$ & $\begin{array}{l}37 \\
(8.1)\end{array}$ & $69(15.1)$ \\
\hline Present study & 40 & 47 & 6 & $3-18(15.1)$ & $28(59.6)$ & $\begin{array}{l}15 \\
(31.9)\end{array}$ & $2(4.3)$ & 0 & $2(4.3)$ & 0 & $3(6.4)$ \\
\hline
\end{tabular}

$N A$ indicates not available; EUS indicates endoscopic ultrasound; FNA indicates fine needle aspiration

and helpful for the clinical management of the patients, supporting the feasibility, safety and validity of EUS in children.

EUS techniques in pediatric still find limited indications, since other validated diagnostic modalities, such as US, CT, MRI or MRCP are more often preferred [14]. However, there is growing evidence (Table 3) to support the role and clinical impact of EUS, particularly to avoid unnecessary ERCP.

In the present study, as in the current literature, the most frequent indication for EUS was the investigation of the pancreatobiliary tract, in particular for suspected CBDs, acute/chronic pancreatitis, and pancreatobiliary abnormality [1, 3, 8, 12-16]. EUS, MRCP, and ERCP are the main diagnostic techniques for pancreatobiliary diseases [6]. For many years, ERCP has been considered the best preoperative diagnostic tool for the examination of the bile duct, although the related complication rate ranges from $5 \%$ to $10 \%$ in adults $[4,9,19]$ and $3.4 \%$ to $28.5 \%$ in children [7]. Regarding the role of endoscopy in the management of suspected choledocholithiasis, the most recent American Society for Gastrointestinal Endoscopy (ASGE) guidelines indicate that clinicians should always perform a non-invasive test, such as EUS or MRCP, before ERCP [4, 6, 9, 19]. Indeed, two systematic reviews showed that MRCP has a high sensitivity ( $85 \%$ to $92 \%$ ) and specificity (93\% to $97 \%$ ) for choledocholithiasis detection [20, 21]. However, EUS has been reported to be the most sensitive and highly specific diagnostic tool for choledocholithiasis and microlithiasis, which are responsible for at least half of all cases of acute pancreatitis. EUS was also found to be more accurate in evaluating microlithiasis of the gallbladder and early chronic/idiopathic pancreatic diseases $[1,3,6,22-$ 28]. In our series, 18 cases presented with suspected biliary stones or acute biliary pancreatitis. EUS revealed CBDs in 2/18 children, who underwent ERCP during the same session. Thus, the EUS approach was helpful to avoid unnecessary ERCP and its associated risks in 16 (88.9\%) patients with imaging suggestive for CBDs.

The therapeutic role of EUS has been clearly demonstrated in the management of pancreatic diseases. Commonly reported indications in children for EUS-FNA are the drainage of pancreatic collections, which is highly helpful in providing a definitive diagnosis $[1,29]$. In the present study, EUS-FNA was performed in 3 patients and allowed a definitive diagnosis in all patients (2 pancreatic masses, 1 pancreatic cyst), who were then 
addressed to appropriate treatment. In the case of a cystic lesion, the cytopathological examination combined with the dosage of tumoral markers permitted a final diagnosis of serous cystadenoma. Traditionally, pancreatic pseudocysts were drained surgically or percutaneously (US or CT guided) [29, 30], but endoscopic drainage became the primary therapeutic modality in the mid-1980s [31]. Moreover, over the last decade, the role of EUS-guided pseudocyst drainage has dramatically increased due to its minimal invasiveness, lower costs, and lower complication rates $[1,32-36]$. In the present case series, the child presenting with a pseudocyst and persistent abdominal pain following acute pancreatitis underwent a successful EUS-guided drainage.

EUS is also a relevant tool in the management of GI pathologies. Indeed, the ability of EUS to differentiate GI wall layers and identify extra-luminal structures makes it the best technique to study mucosal/submucosal lesions observed during conventional endoscopy $[12,29]$. In the present study, EUS allowed the precise definition of the invasion of the muscularis layer in a patient with duodenal NET, preventing a non-radical endoscopic resection in favor of an adequate surgical treatment.

Regarding the application in the lower GI tract, EUS plays a major role in rectal cancer staging in the adult population [37, 38]. In children, EUS has been mainly used to evaluate anorectal anomalies, anal sphincter defects, and anal Crohn's disease [3, 39]. In the present series, as in the previous literature [17, 40], EUS examination was found to be very precise in describing anorectal normal and abnormal anatomy, which guided the subsequent medical/surgical management. EUS was also useful in the follow-up period to evaluate the response to Crohn's disease therapy. It must be noted, however, that the most common imaging modalities for the evaluation of anorectal anatomy remain CT and pelvic MRI. Both of these techniques have drawbacks: CT is associated with radiation exposure while MRI application is limited by high costs and restricted access in many centers $[39,41]$. Moreover, in very young children, these methods require sedation. Conversely, EUS has the advantage that it may be performed at the same time as colonoscopy by a gastroenterologist, who can interpret both the clinical and imagery observations simultaneously and perform ERCP during the same session, if needed [17]. However, the final choice of which imaging modality to apply currently remains mainly dependent on institutional resources and clinical expertise.

The present study has some limitations. First, it is a retrospective analysis of data from a single high-volume center. The sample size is relatively small, with younger children and infants not adequately represented; indeed, the majority of the patients treated and evaluated were adolescents, limiting the possibility to generalize results to other ages. Finally, the paucity of EUS-FNA procedures performed does not allow the drawing of definitive conclusions.

Currently, the use of EUS in children is limited by the low availability of echoendoscopes in most pediatric centers together with the scarce experience and training of most pediatric gastroenterologists. In the near future, it is advisable that pediatric gastroenterologists acquire a specific expertise with EUS to extend the use of this diagnostic and therapeutic technique in pediatric populations.

\section{Conclusion}

This single center case series supports the applicability, feasibility, and safety of EUS and EUS-FNA in the management of pediatric pancreatobiliary and GI disorders. Further research and large-scale studies are needed to standardize the indications and applications for EUS in pediatric populations.

\section{Abbreviations}

ASA: American Society of Anesthesiologists; CBDs: Common bile duct stones; CT: Computed tomography; ERCP: Endoscopic retrograde

cholangiopancreatography; EUS: Endoscopic ultrasound; FNA: Fine needle aspiration; Gl: Gastrointestinal; MRCP: Magnetic resonance

cholangiopancreatography; MRI: Magnetic resonance imaging;

NET: Neuroendocrine tumor; US: Ultrasound

\section{Acknowledgements}

The authors would like to thank the medical and nursing staff of the Gastroenterology and Endoscopy Unit of the University Hospital of Parma for helping making this study possible.

Funding

none.

Availability of data and materials

The datasets during and/or analyzed during the current study is available from the corresponding author on reasonable request.

\section{Authors' contributions}

$A F, B B$, and GLdeA participated in the patients' treatment, study design, and manuscript drafting; AF and FG contributed to the literature search, data collection, and data analysis; AG, FG and MM were involved in the patients' follow-up and data collection; PC performed the cytological analysis; MCC, NdeA, and GLdeA critically revised the manuscript and substantially contributed to the final version of the manuscript. All authors read and approved the final manuscript.

\section{Ethics approval and consent to participate}

Written consent was obtained from both parents or legal guardians, and it included consent for the therapeutic procedures. All data were collected in compliance with the ethical principles stated in the Declaration of Helsinki and according to the Good Clinical Practice protocols and Privacy Protection Law of the institution.

\section{Consent for publication}

Patients and their parents or guardians have provided permission to publish these data, and their identity has been protected.

\section{Competing interests}

The authors declare that they have no conflicts of interest regarding the publication of this paper. 


\section{Publisher's Note}

Springer Nature remains neutral with regard to jurisdictional claims in published maps and institutional affiliations.

\begin{abstract}
Author details
${ }^{1}$ Gastroenterology and Endoscopy Unit, University Hospital of Parma, Via Gramsci 14, 43126 Parma, Italy. "Department of Pediatrics, "Pietro Barilla" Children's Hospital, University Hospital of Parma, Via Gramsci 14, 43126 Parma, Italy. ${ }^{3}$ Department of Pathology, University Hospital of Parma, 43126 Parma, Italy. ${ }^{4}$ University Paris VII, Rothschild Hospital, AP-HP, Paris, France. ${ }^{5}$ Unit of Digestive, Hepato-Pancreato-Biliary Surgery and Liver Transplantation, Henri Mondor Hospital, AP-HP, 94010 Paris, Créteil, France. ${ }^{6}$ Cancer Research Lab. EC2M3, Université Paris-Est, Créteil, Val de Marne UPEC, 94010 Paris, France.
\end{abstract}

Received: 2 August 2016 Accepted: 28 November 2017 Published online: 06 December 2017

\section{References}

1. Scheers I, Ergun M, Aouattah T, Piessevaux H, Borbath I, Stephenne X, De Magnee C, Reding R, Sokal E, Veyckemans F, et al. Diagnostic and therapeutic roles of endoscopic ultrasound in pediatric Pancreaticobiliary disorders. J Pediatr Gastroenterol Nutr. 2015;61(2):238-47.

2. Sharma V, Rana SS, Bhasin DK. Endoscopic ultrasound guided interventional procedures. World J Gastrointest Endosc. 2015;7(6):628-42.

3. Cohen S, Kalinin M, Yaron A, Givony S, Reif S, Santo E. Endoscopic ultrasonography in pediatric patients with gastrointestinal disorders. J Pediatr Gastroenterol Nutr. 2008;46(5):551-4.

4. Fabbri C, Polifemo AM, Luigiano C, Cennamo V, Fuccio L, Billi P, Maimone A, Ghersi S, Macchia S, Mwangemi C, et al. Single session versus separate session endoscopic ultrasonography plus endoscopic retrograde cholangiography in patients with low to moderate risk for choledocholithiasis. J Gastroenterol Hepatol. 2009;24(6):1107-12

5. Committee ASoP, Lightdale JR, Acosta R, Shergill AK, Chandrasekhara V, Chathadi K, Early D, Evans JA, Fanelli RD, Fisher DA, et al. Modifications in endoscopic practice for pediatric patients. Gastrointest Endosc. 2014;79(5):699-710.

6. Committee ASoP, Maple JT, Ben-Menachem T, Anderson MA, Appalaneni V, Banerjee S, Cash BD, Fisher L, Harrison ME, Fanelli RD, et al. The role of endoscopy in the evaluation of suspected choledocholithiasis. Gastrointest Endosc. 2010;71(1):1-9.

7. Kieling CO, Hallal C, Spessato CO, Ribeiro LM, Breyer H, Goldani HA, Maguilnik I. Changing pattern of indications of endoscopic retrograde cholangiopancreatography in children and adolescents: a twelve-year experience. World J Pediatr. 2015;11(2):154-9.

8. Mahajan R, Simon EG, Chacko A, Reddy DV, Kalyan PR, Joseph AJ, Dutta AK, Chowdhury SD, Kurien RT. Endoscopic ultrasonography in pediatric patients-experience from a tertiary care center in India. Indian J Gastroenterol. 2016:35(1):14-9.

9. Anderloni A, Galeazzi M, Ballare M, Pagliarulo M, Orsello M, Del Piano M, Repici A. Early endoscopic ultrasonography in acute biliary pancreatitis: a prospective pilot study. World J Gastroenterol. 2015;21(36):10427-34.

10. Vazquez-Sequeiros E, Gonzalez-Panizo Tamargo F, Boixeda-Miquel D, Milicua JM. Diagnostic accuracy and therapeutic impact of endoscopic ultrasonography in patients with intermediate suspicion of choledocholithiasis and absence of findings in magnetic resonance cholangiography. Rev Esp Enferm Dig. 2011;103(9):464-71.

11. Gordon K, Conway J, Evans J, Petty J, Fortunato JE, Mishra G. EUS and EUS guided interventions Alter clinical Management in Children with digestive diseases. J Pediatr Gastroenterol Nutr. 2015:

12. Al-Rashdan A, LeBlanc J, Sherman S, McHenry L, DeWitt J, Al-Haddad M. Role of endoscopic ultrasound for evaluating gastrointestinal tract disorders in pediatrics: a tertiary care center experience. J Pediatr Gastroenterol Nutr. 2010;51(6):718-22.

13. Attila T, Adler DG, Hilden K, Faigel DO. EUS in pediatric patients. Gastrointest Endosc. 2009;70(5):892-8.

14. Roseau G, Palazzo L, Dumontier I, Mougenot JF, Chaussade S, Navarro J, Couturier D. Endoscopic ultrasonography in the evaluation of pediatric digestive diseases: preliminary results. Endoscopy. 1998;30(5):477-81.

15. Varadarajulu S, Wilcox CM, Eloubeidi MA. Impact of EUS in the evaluation of pancreaticobiliary disorders in children. Gastrointest Endosc. 2005;62(2):239-44.
16. Bjerring OS, Durup J, Qvist N, Mortensen MB. Impact of upper gastrointestinal endoscopic ultrasound in children. J Pediatr Gastroenterol Nutr. 2008;47(1):110-3.

17. Rosen MJ, Moulton DE, Koyama T, Morgan WM 3rd, Morrow SE, Herline AJ, Muldoon RL, Wise PE, Polk DB, Schwartz DA. Endoscopic ultrasound to guide the combined medical and surgical management of pediatric perianal Crohn's disease. Inflamm Bowel Dis. 2010;16(3):461-8.

18. Bosman FT, World Health Organization, International Agency for Research on Cancer. WHO classification of tumours of the digestive system. In: Lyon: International Agency for Research on Cancer. 4th ed; 2010.

19. Kwon Cl, Song SH, Hahm KB, Ko KH. Unusual complications related to endoscopic retrograde cholangiopancreatography and its endoscopic treatment. Clin Endosc. 2013;46(3):251-9.

20. Romagnuolo J, Bardou M, Rahme E, Joseph L, Reinhold C, Barkun AN. Magnetic resonance cholangiopancreatography: a meta-analysis of test performance in suspected biliary disease. Ann Intern Med. 2003;139(7): 547-57.

21. Verma D, Kapadia A, Eisen GM, Adler DG. EUS vs MRCP for detection of choledocholithiasis. Gastrointest Endosc. 2006;64(2):248-54.

22. Anderloni A, Repici A. Role and timing of endoscopy in acute biliary pancreatitis. World J Gastroenterol. 2015;21(40):11205-8.

23. Karakan T, Cindoruk M, Alagozlu H, Ergun M, Dumlu S, Unal S. EUS versus endoscopic retrograde cholangiography for patients with intermediate probability of bile duct stones: a prospective randomized trial. Gastrointest Endosc. 2009;69(2):244-52

24. Tse F, Liu L, Barkun AN, Armstrong D, Moayyedi P. EUS: a meta-analysis of test performance in suspected choledocholithiasis. Gastrointest Endosc. 2008;67(2):235-44.

25. Wilcox CM, Varadarajulu S, Eloubeidi M. Role of endoscopic evaluation in idiopathic pancreatitis: a systematic review. Gastrointest Endosc. 2006;63(7): 1037-45.

26. Catalano MF, Lahoti S, Geenen JE, Hogan WJ. Prospective evaluation of endoscopic ultrasonography, endoscopic retrograde pancreatography, and secretin test in the diagnosis of chronic pancreatitis. Gastrointest Endosc. 1998;48(1):11-7.

27. Liu CL, Lo CM, Chan JK, Poon RT, Lam CM, Fan ST, Wong J. Detection of choledocholithiasis by EUS in acute pancreatitis: a prospective evaluation in 100 consecutive patients. Gastrointest Endosc. 2001;54(3): 325-30.

28. Anderloni A, Ballare M, Pagliarulo M, Conte D, Galeazzi M, Orsello M, Andorno S, Del Piano M. Prospective evaluation of early endoscopic ultrasonography for triage in suspected choledocholithiasis: results from a large single centre series. Dig Liver Dis. 2014;46(4):335-9.

29. Godfrey EM, Rushbrook SM, Carroll NR. Endoscopic ultrasound: a review of current diagnostic and therapeutic applications. Postgrad Med J. 2010; 86(1016):346-53.

30. Jazrawi SF, Barth BA, Sreenarasimhaiah J. Efficacy of endoscopic ultrasoundguided drainage of pancreatic pseudocysts in a pediatric population. Dig Dis Sci. 2011;56(3):902-8.

31. Kozarek RA, Brayko CM, Harlan J, Sanowski RA, Cintora I, Kovac A. Endoscopic drainage of pancreatic pseudocysts. Gastrointest Endosc. 1985: 31(5):322-7.

32. Chevaux JB, Deprez PH. Established EUS-guided therapeutic interventions. Minerva Med. 2014;105(5):333-51.

33. Giovannini M, Binmoeller K, Seifert H. Endoscopic ultrasound-guided cystogastrostomy. Endoscopy. 2003;35(3):239-45.

34. Habashi S, Draganov PV. Pancreatic pseudocyst. World J Gastroenterol. 2009; 15(1):38-47.

35. Wang S, Liu W, Sun S, Liu X, Wang S, Ge N, Wang G, Guo J. Endoscopic ultrasonography-guided drainage of a pancreatic pseudocyst one week after formation. Endosc Ultrasound. 2015;4(3):271-2.

36. Anderloni A, Orellana F, Jovani M, Repici A. Endoscopic ultrasound-guided drainage of a pancreatic pseudocyst with a novel lumen-apposing metal stent on an electrocautery-enhanced delivery system. Dig Liver Dis. 2015; 47(10):e17.

37. Cho JW. The role of Endosonography in the staging of gastrointestinal cancers. Clin Endosc. 2015;48(4):297-301.

38. Committee ASoP, Fisher DA, Shergill AK, Early DS, Acosta RD, Chandrasekhara V, Chathadi KV, Decker GA, Evans JA, Fanelli RD, et al. Role of endoscopy in the staging and management of colorectal cancer. Gastrointest Endosc. 2013;78(1):8-12. 
39. Wang Z, Hu L, Jin X, Li X, Xu L. Evaluation of postoperative anal functions using endoanal ultrasonography and anorectal manometry in children with congenital anorectal malformations. J Pediatr Surg. 2016;51(3):416-20.

40. Roseau G, Palazzo L. Anorectal echo-endoscopy: applications in pediatrics. Presse Med. 1999;28(35):1946-8.

41. Morinville VD, Paquin SC, Sahai AV. Safety, feasibility, and usefulness of rectal endoscopic ultrasonography for pediatric anal and perianal complaints. J Pediatr Gastroenterol Nutr. 2010;51(1):93-5.

Submit your next manuscript to BioMed Central and we will help you at every step:

- We accept pre-submission inquiries

- Our selector tool helps you to find the most relevant journal

- We provide round the clock customer support

- Convenient online submission

- Thorough peer review

- Inclusion in PubMed and all major indexing services

- Maximum visibility for your research

Submit your manuscript at www.biomedcentral.com/submit 